\title{
Search for Roma-BZCAT Blazars in the Palermo BAT Survey archive
}

\author{
B. Sbarufatti* ${ }^{a}$ V. La Parola, G. Cusumano, V. Mangano, A. Segreto, P. Romano \\ INAF-IASFPa \\ Via U. La Malfa 153 \\ I-90146 Palermo \\ Italy \\ ${ }^{a}$ E-mail: sbarufatti@ifc.inaf.it
}

\section{S. Campana, G. Chincarini, G. Tagliaferri}

INAF-OAB

via E. Bianchi 46

I-23807 Merate

Italy

\section{P. Giommi}

$A S D C$

via Galileo Galilei

I-00044 Frascati

Italy

\begin{abstract}
Blazars are the most numerous class of extragalactic $\gamma$-ray sources. Because of their very broad spectral emission, blazars are expected to play an important role in the generation of the extragalactic background in several energy bands. Using the Palermo BAT survey archive we searched for the hard X-ray counterparts of the $\sim 2600$ objects in the Roma-BZCAT Blazars catalogue to study their contribution to the $15-150 \mathrm{keV}$ extragalactic emission.
\end{abstract}

7th INTEGRAL Workshop

September 8-11 2008

Copenhagen, Denmark

${ }^{*}$ Speaker. 


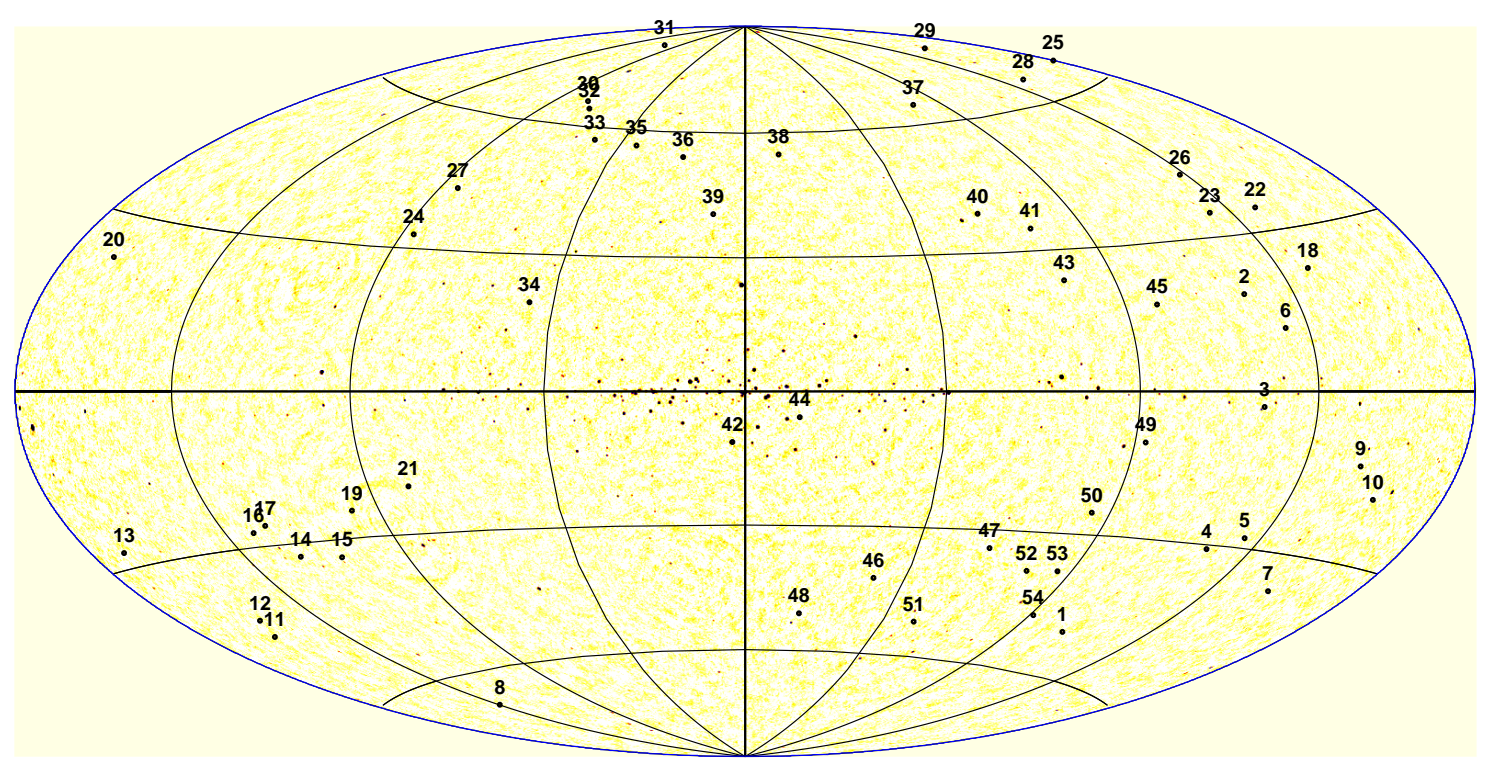

Figure 1: Palermo BAT Survey map of the sky in galactic coordinates. Blazars from the Roma-BZCAT catalogue detected above a $4.8 \sigma$ level in the Palermo BAT Survey are shown.

The BAT survey archive consists of data collected by the Burst Alert Telescope [1] on board of the Swift spacecraft [2] while the observatory waits for Gamma ray Bursts (GRBs). Our team is exploiting observations from the first 39 months of the Swift mission through a systematic search for new hard X-ray sources by means of the BatImager [ $₫$, a dedicated software, completely independent from the one developed by the Swift-BAT hardware team, to process the BAT survey data.

The Roma-BZCAT (http://www.asdc.asi.it/bzcat) is a catalogue of blazars based on multifrequency surveys and an extensive review of the literature ([3]). It contains $\sim 2600$ sources, classified either as BL Lac objects, Flat Spectrum Radio Quasars and blazars of uncertain/unknown nature. We searched for the counterparts of these sources in the BAT survey with the BatImager, processing the whole sky significance map and estimating rates and significances in the locations of the Roma-BZCAT blazars. We considered a source detected if its significance was above $4.8 \sigma$ in at least one of the considered energy bands (15-150 keV, 15-30 keV, 15-70 keV). We found a significant hard X-ray counterpart for 54 Roma-BZCAT sources:

- 14 BL Lac objects;

\section{- 32 FSRQ;}

- 8 unclassified blazars.

In Fig. 11 we show the location of the detected sources in Palermo BAT survey mosaic image of the whole sky. Comparing these detections with the ones we obtained from a list of $\sim 2600$ random positions on the sky, we estimate that there is a $10 \%$ chance that one of our sources is due to spurious association. The source properties (name, position, count rates and significance of detection in the $15-150 \mathrm{keV}, 15-30 \mathrm{keV}$ and $15-70 \mathrm{keV}$ energy bands) are reported in Table 1 . 


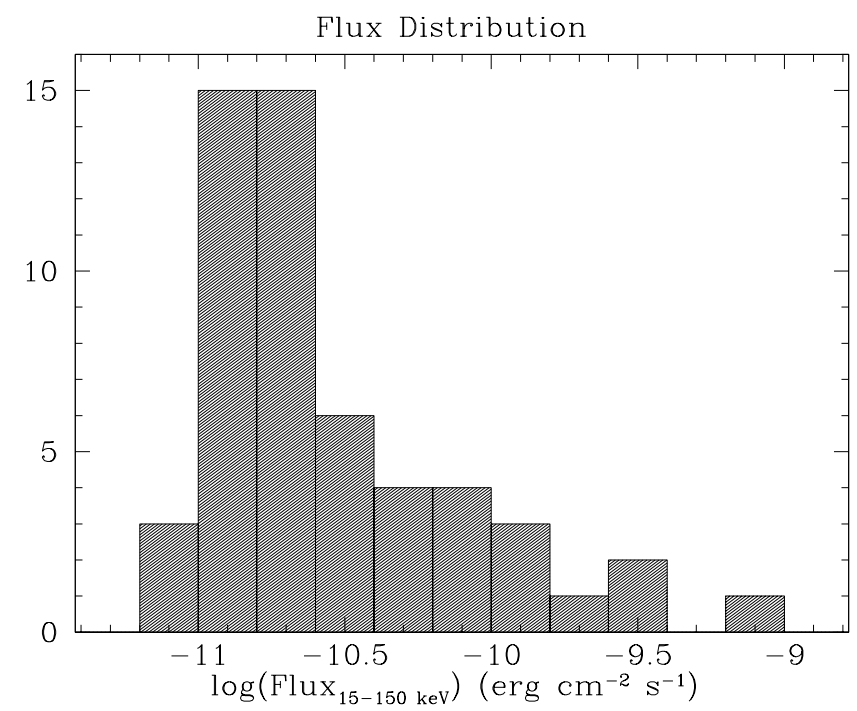

Figure 2: Flux distribution for Roma-BZCAT blazars detected in the BAT survey.

The flux distribution of the detected sources is reported in Fig. 2. We detect sources in the $10^{-11}-10^{-9} \mathrm{ergs} \mathrm{cm}^{-2} \mathrm{~s}^{-1}$ range.

In Fig. 3 we show the hardness ratios (30-70/15-30 keV vs. $150-70 / 15-30 \mathrm{keV})$. The distribution of BL Lac objects and FSRQ have no significant differences in this plane.

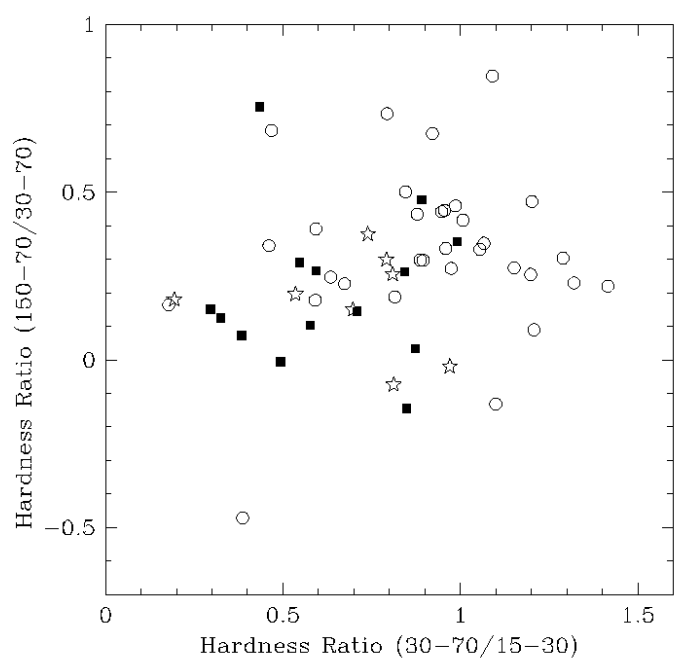

Figure 3: Hardness ratios for for Roma-BZCAT blazars detected in the BAT survey. Filled squares indicate FSRQ, open circles indicate BL Lac objects and stars indicate unclassified sources.

Searching in the literature, we were able to find a determination of the redshift for 53 sources. We show the redshift distribution in Fig. $\emptyset$. As expected, most of the sources are at relatively low redshift, but we detect blazars up to $\mathrm{z} \sim 3.5$. The inferred luminosity distribution is shown in Fig. 5. We observe luminosities in the $10^{42}-10^{48} \mathrm{ergs} \mathrm{s}^{-1}$ range, with more than $50 \%$ of the objects above $10^{46} \mathrm{ergs} \mathrm{s}^{-1}$.

In order to better constrain the properties of these objects, we plan to make use of data in other 
bands of the electromagnetic spectrum, already existing in the literature or in the archives, or to be obtained in future observations. In particular, we will check for differences in the observed properties of the various blazar subclasses (HBL, LBL, IBL, FSRQ) in the BAT energy band and if and how these properties correlate with observations in other bands of the electromagnetic spectrum.

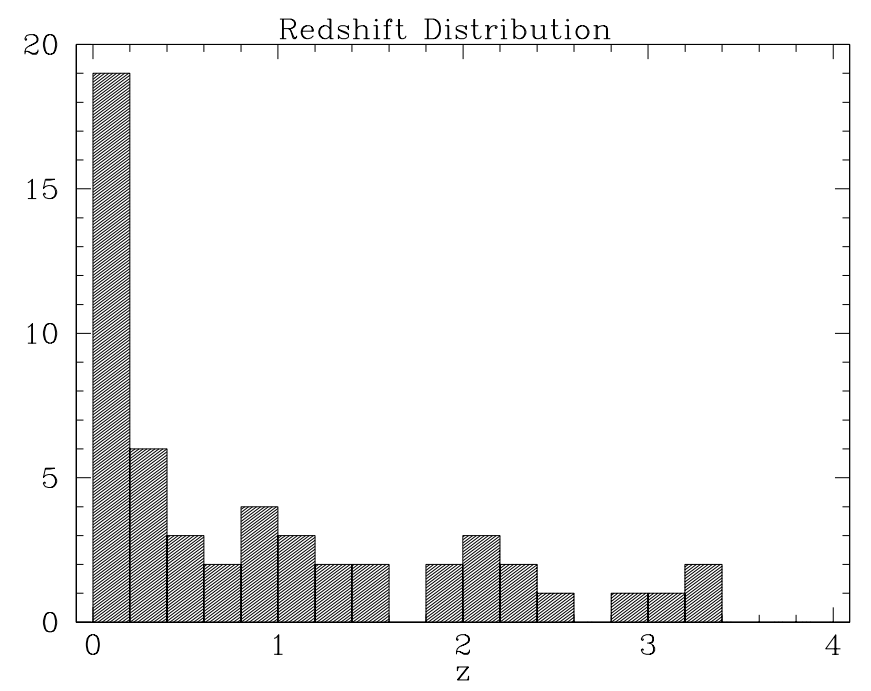

Figure 4: Redshift distribution for Roma-BZCAT blazars detected in the BAT survey.

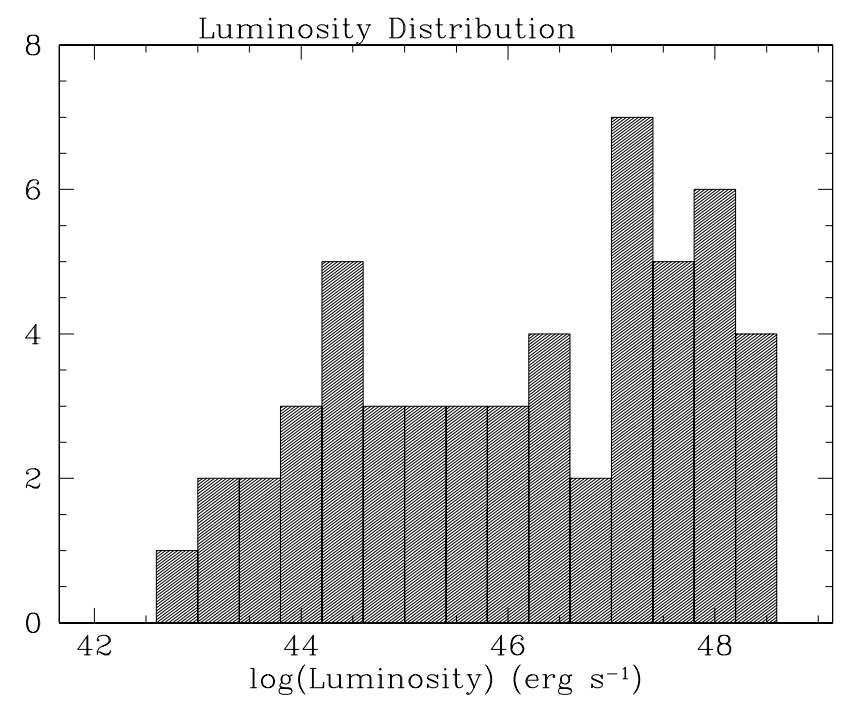

Figure 5: Luminosity distribution for Roma-BZCAT blazars detected in the BAT survey. 
Table 1: Roma-BZCAT blazars detected in the BAT survey.

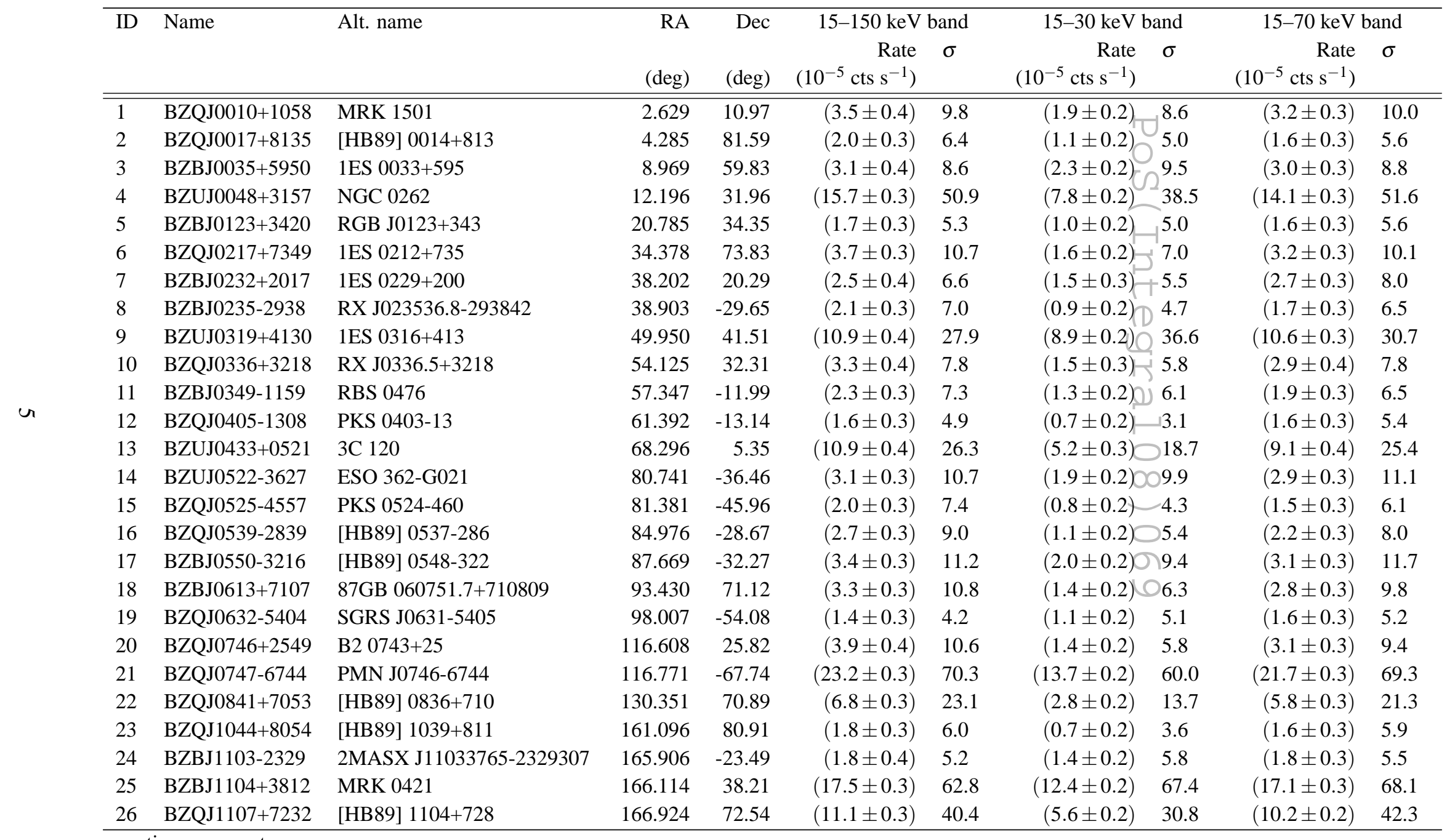




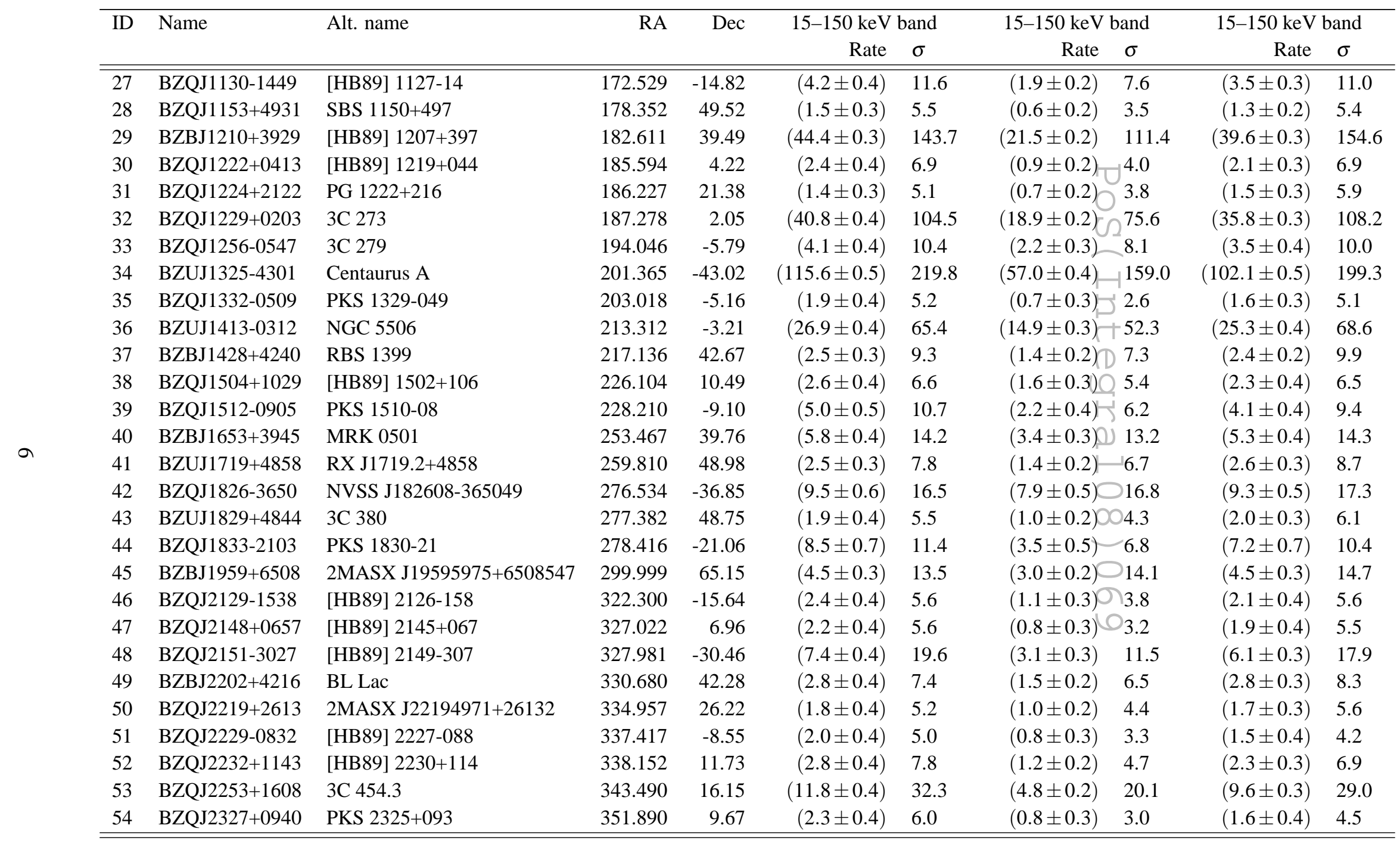




\section{Acknowledgments}

This work is supported at INAF by funding from ASI trough grant I/088/06/0 and by PRIN MIUR grant CRA 1.06.10.07. Italian researchers acknowledge the support of Nature (455, 835836) and thanks the Editors for increasing the international awareness of the current critical situation of the Italian Research.

\section{References}

[1] Barthelmy, S. D., et al., 2005, SSRv, 120, 143

[2] Gehrels, N., et al., 2004, ApJ, 611, 1005

[3] Massaro, E.et al., 2008, MmSAI, 79, 262

[4] Segreto, A., et al., 2008, in preparation 